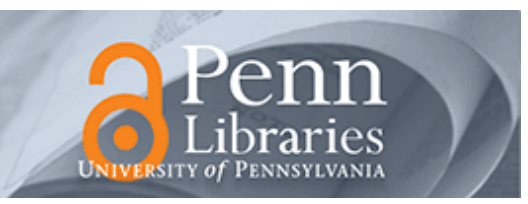

University of Pennsylvania

ScholarlyCommons

$1-12-2009$

\title{
Simulations and electrical conductivity of percolated networks of finite rods with various degrees of axial alignment
}

\author{
Sadie I. White \\ University of Pennsylvania, whitesa@seas.upenn.edu \\ Brian A. DiDonna \\ Stellar Science \\ Minfang Mu \\ University of Pennsylvania, minfang@seas.upenn.edu \\ Tom C. Lubensky \\ University of Pennsylvania, tom@physics.upenn.edu \\ Karen I. Winey \\ University of Pennsylvania, winey@seas.upenn.edu
}

Follow this and additional works at: https://repository.upenn.edu/mse_papers

\section{Recommended Citation}

White, S. I., DiDonna, B. A., Mu, M., Lubensky, T. C., \& Winey, K. I. (2009). Simulations and electrical conductivity of percolated networks of finite rods with various degrees of axial alignment. Retrieved from https://repository.upenn.edu/mse_papers/163

Simulations and electrical conductivity of percolated networks of finite rods with various degrees of axial alignment Sadie I. White, Brian A. DiDonna, Minfang Mu, T. C. Lubensky, and Karen I. Winey, Phys. Rev. B 79, 024301 (2009), DOI:10.1103/PhysRevB.79.024301

Copyright American Physical Society.

Reprinted from Physical Review B, Volume 79, Issue 2, Article 024301, January 2009.

Publisher URL: http://link.aps.org/doi/10.1103/PhysRevB.79.024301

This paper is posted at ScholarlyCommons. https://repository.upenn.edu/mse_papers/163

For more information, please contact repository@pobox.upenn.edu. 


\title{
Simulations and electrical conductivity of percolated networks of finite rods with various degrees of axial alignment
}

\author{
Abstract \\ We present a three-dimensional simulation and calculation of electrical conductivity above the filler \\ percolation threshold for networks containing finite, conductive cylinders as a function of axial orientation \\ (S) and aspect ratio (L/D). At a fixed volume fraction and L/D, the simulations exhibit a critical degree of \\ orientation, S-C, above which the electrical conductivity decreases dramatically. With increasing filler \\ concentration and aspect ratio, this critical orientation shifts to higher degrees of alignment. Additionally, \\ at a fixed volume fraction and L/D, the simulated electrical conductivity displays a maximum at slight \\ uniaxial orientation, which is less pronounced at higher volume fractions and aspect ratios. Our approach \\ can be used as a predictive tool to design the optimal filler concentration and degree of orientation \\ required to maximize electrical conductivity in polymer nanocomposites with conductive cylindrical fillers \\ of finite dimension.

\section{Keywords} \\ CONTINUUM PERCOLATION, STICKS

\section{Comments} \\ Simulations and electrical conductivity of percolated networks of finite rods with various degrees of axial \\ alignment Sadie I. White, Brian A. DiDonna, Minfang Mu, T. C. Lubensky, and Karen I. Winey, Phys. Rev. B \\ 79, 024301 (2009), DOI:10.1103/PhysRevB.79.024301 \\ Copyright American Physical Society. \\ Reprinted from Physical Review B, Volume 79, Issue 2, Article 024301, January 2009. \\ Publisher URL: http://link.aps.org/doi/10.1103/PhysRevB.79.024301
}




\title{
Simulations and electrical conductivity of percolated networks of finite rods with various degrees of axial alignment
}

\author{
Sadie I. White, ${ }^{1}$ Brian A. DiDonna, ${ }^{2}$ Minfang Mu, ${ }^{1}$ T. C. Lubensky, ${ }^{3}$ and Karen I. Winey ${ }^{1, *}$ \\ ${ }^{1}$ Department of Materials Science and Engineering, University of Pennsylvania, Philadelphia, Pennsylvania 19104, USA \\ ${ }^{2}$ Stellar Science, Suite 725, 6565 America's Parkway NE, Albuquerque, New Mexico 87110, USA \\ ${ }^{3}$ Department of Physics and Astronomy, University of Pennsylvania, Philadelphia, Pennsylvania 19104, USA
}

(Received 22 August 2008; published 12 January 2009)

\begin{abstract}
We present a three-dimensional simulation and calculation of electrical conductivity above the filler percolation threshold for networks containing finite, conductive cylinders as a function of axial orientation $(S)$ and aspect ratio $(L / D)$. At a fixed volume fraction and $L / D$, the simulations exhibit a critical degree of orientation, $S_{c}$, above which the electrical conductivity decreases dramatically. With increasing filler concentration and aspect ratio, this critical orientation shifts to higher degrees of alignment. Additionally, at a fixed volume fraction and $L / D$, the simulated electrical conductivity displays a maximum at slight uniaxial orientation, which is less pronounced at higher volume fractions and aspect ratios. Our approach can be used as a predictive tool to design the optimal filler concentration and degree of orientation required to maximize electrical conductivity in polymer nanocomposites with conductive cylindrical fillers of finite dimension.
\end{abstract}

DOI: 10.1103/PhysRevB.79.024301

PACS number(s): 72.80.Tm

\section{INTRODUCTION}

We present a three-dimensional simulation and calculation of electrical conductivity above the filler percolation threshold for networks containing conductive, finite-sized rods as a function of axial orientation. Recent advances in materials chemistry have produced a variety of electrically conductive particles, including carbon nanotubes and metal nanowires, which have modest aspect ratios $(L / D \sim 10-80)$. When these particles are fabricated into composites or mats, the slightest flow or external field produces an anisotropic particle orientation. The extent of orientation, as well as the aspect ratio and concentration, influences the connectivity in the network of particles even above the percolation threshold. This paper presents simulation results for cylindrical rods with aspect ratios of 10, 20, and 80 and a distribution of axial orientations. After the rod network is generated, the random resistor network model is used to calculate the electrical conductivity of the network. As the degree of axial orientation increases, the electrical conductivity initially increases and then dramatically decreases as the rods become more highly aligned. This dependence of axial orientation is most pronounced at lower volume fractions of rods and for rods with lower aspect ratios. These simulated results qualitatively agree with recent electrical conductivity measurements of polymer nanocomposites containing carbon nanotubes with $L / D \sim 45$. Natural extensions of our rod simulation coupled with an electrical conductivity calculation include exploring the relative importance of aspect ratio, size dispersity, rod-rod contact resistance, and alignment anisotropy in maximizing electrical conductivity.

Percolation behavior has been extensively investigated since the mid-1950s both theoretically and experimentally. Percolation models are used to describe the rapid transition from one behavior to another, and they have been successfully applied to the onset of high electrical conductivity in composites consisting of conductive fillers in insulating matrices. Early percolation models ${ }^{1}$ use lattices where particles (typically spheres) are confined to periodic positions. Continuum percolation models do not require a lattice and are more realistic for materials with discrete filler particles. The primary focus of lattice and continuum models has been the prediction of the onset of percolation as a function of filler content. In the case of nonspherical fillers, the percolation threshold has been examined with simulations and analytical models in two dimensions ${ }^{2-5}$ and three dimensions ${ }^{3,6-11}$ as a function of filler orientation. To date, studying the properties of networks above the percolation threshold has been of secondary importance. However, the increasing availability of functional nanoparticles of finite aspect ratio motivates not only the prediction of the percolation threshold but also the properties (e.g., electrical conductivity) of networks above the percolation threshold.

\section{EXPERIMENTAL}

Our simulations used a rectangular prism $(l=1$ unit, $w$ $=\sqrt{ } 0.1$ unit, and $h=\sqrt{ } 0.1$ unit $)$ that was populated sequentially with soft-core (i.e., interpenetrable) cylindrical particles with length of 0.04 unit and diameters of 0.0005 , 0.002 , or 0.004 unit to give aspect ratios $(L / D)$ of 80,20 , and 10 , respectively. Two rods were determined to be in contact when the minimum distance between their long axes was less than 1 rod diameter. Particle-particle contacts were identified after each new cylinder was added and were used to define clusters of overlapping rods. Percolation is defined as the presence of a cluster of contacting rods that spans the long axis of the simulation volume. Periodic boundary conditions were used.

In all cases, the centers of mass of the rods were randomly distributed in the rectangular prism. For the isotropic simulations, the angular distributions of the rod axes were uniformly random. The isotropic volume percolation threshold, $\phi_{c, \text { iso }}$, was determined for each aspect ratio by multiplying the number of rods required to achieve percolation by the volume of one rod. For the nonisotropic simulations, the vol- 
a

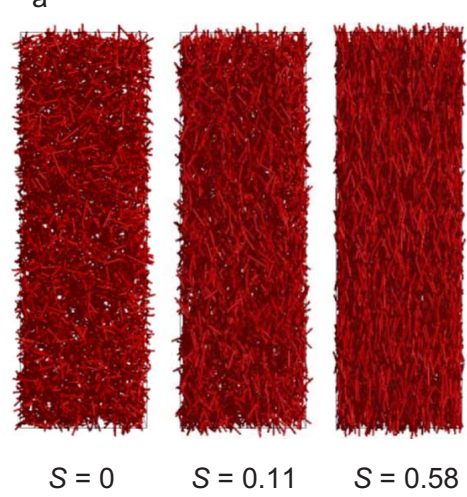

b

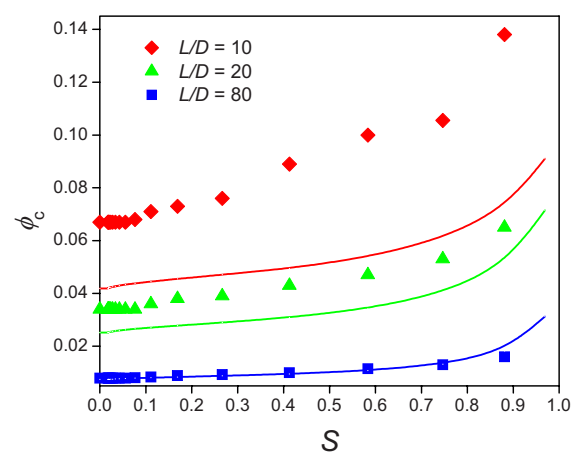

FIG. 1. (Color online) (a) Simulated morphologies of finite rods having different amounts of axial alignment. The axial orientation parameter, $S$, is defined as $S=\left(3\left\langle\cos ^{2} \theta\right\rangle-1\right) / 2$ and varies from 0 to 1 corresponding to isotropic and perfectly aligned rods, respectively. The simulation volume is 1 unit long with a cross-sectional area of 0.1 unit $^{2}$. These simulated rods have an $L / D=10$ and volume fractions of $0.075,0.082$, and 0.118 for $S$ of $0,0.11$, and 0.58 , respectively. The electrical conductivity of these simulated morphologies was calculated using the random resistor network model. (b) The critical volume fraction, $\phi_{c}$, was determined at fixed $S$ using a power law to relate electrical conductivity and volume fraction for three values of $L / D$. For comparison, $\phi_{c}$ from the excluded volume model [Eq. (4)] is shown as a line and notable discrepancies are observed at lower $L / D$.

ume was populated with the same random distribution of rod centers but with a Gaussian distribution in $\cos \theta$ centered around $\theta=0$, where $\theta$ is the angle between the rod axis and the long $(z)$ axis of the simulation volume. The extent of alignment is defined by the axial orientation parameter, $S=\left(3\left\langle\cos ^{2} \theta\right\rangle-1\right) / 2$, where $S$ varies from 0 , for isotropically oriented rods, to 1 , for perfectly aligned rods [Fig. 1(a)]. The simulations were performed at a range of rod volume fractions, $\phi$, corresponding to $\sim 1500-100000$ rods.

The electrical conductivity of each simulated sample was calculated using the random resistor network model. ${ }^{1}$ The internal resistances of the matrix and the rods were set to infinity and zero, respectively. Each rod-rod contact (node) was set at a resistance of $1 \Omega$, and the voltage drop across the long axis (i.e., the alignment axis) of the sample was $1 \mathrm{~V}$. Kirchoff's law was used to balance the current flow through each node. ${ }^{1}$ The conductivity was calculated along the long axis of each simulation prism by measuring the simulated
TABLE I. Comparison of $\phi_{c \text {,iso }}$ from our simulations and two variations in the excluded volume model.

\begin{tabular}{llll}
\hline \hline$L / D$ & $\phi_{c, \text { iso }}$ & $\phi_{c, \text { iso }}^{\text {exvol }}$ & $\phi_{c, \text { iso }}^{\text {contact }}$ \\
\hline 10 & 0.072 & 0.042 & 0.060 \\
20 & 0.036 & 0.025 & 0.030 \\
80 & 0.0081 & 0.0075 & 0.0075 \\
\hline \hline
\end{tabular}

current through the sample using $\sigma=(I l) /(V A)$, where $l$ is the length of the sample (1 unit) and $A$ is its cross-sectional area $\left(0.1\right.$ unit $\left.^{2}\right)$. Each set of simulation conditions was repeated 100 times, and the average electrical conductivity values are reported.

\section{RESULTS AND DISCUSSION}

We first compare our percolation results for isotropic networks to two established models (Table I). Our simulations

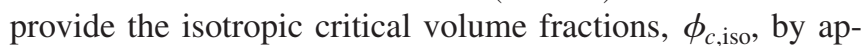
plying a power law to the calculated electrical conductivities at $S=0$,

$$
\sigma_{\mathrm{el}} \propto\left(\frac{\phi_{c, \text { iso }}-\phi}{\phi_{c, \text { iso }}}\right)^{\beta} .
$$

The excluded volume model is the most commonly used continuum model for the description of the onset of percolation. ${ }^{6-10}$ This model has been applied to filler particles of all shapes and describes the volume around a filler object into which the center of mass of a second, identical object cannot enter without contacting the first object. The excluded volume of a soft-core interpenetrable cylinder is

$$
V_{\mathrm{ex}}=8 \pi L R^{2}+4 L^{2} R\langle|\sin \gamma|\rangle,
$$

where $L$ is the length of the cylinder, $R$ is its radius, and $\gamma$ is the angle between two rods. The excluded volume is related to the number of fillers required for percolation $\left(N_{c}\right)$ by

$$
N_{c} \cong \frac{V}{V_{\mathrm{ex}}},
$$

where $V$ is the total sample volume. In the slender-rod limit (i.e., as $L / 2 R$ approaches infinity), this relation becomes a true equality. For isotropic rods, $\langle|\sin \gamma|\rangle=\pi / 4$ so that $\phi_{c \text {,iso }}^{\text {exvol }}$ for soft-core cylinders is

$$
\phi_{c, \text { iso }}^{\text {exvol }}=\frac{N_{c}\left(\pi R^{2} L\right)}{V}=\frac{R}{(8 R+L)} .
$$

Both $\phi_{c \text {,iso }}$ and $\phi_{c \text {,iso }}^{\text {exvol }}$ decrease as the aspect ratio increases, but the excluded volume model systematically predicts a smaller percolation threshold in an isotropic network than our simulations. This discrepancy increases at smaller aspect ratios, thereby highlighting the importance of our simulations of rods with modest aspect ratios.

Other approaches to predicting percolation behavior have combined simulations and excluded volume models. By using a Monte Carlo simulation in which soft-core rods were placed into a unit volume with random orientations, Foygel 
et $a l .{ }^{10}$ determined $\phi_{c \text {, iso }}^{\text {contact }}$ by counting the number of rods required to generate a percolation cluster. The number of contacts per rod $\left(B_{c}\right)$ at the onset of percolation, which is not considered in the traditional excluded volume model, was then found by fitting to the following equation:

$$
\phi_{c, \text { iso }}^{\text {contact }}=B_{c} N_{c} \frac{V_{1 \mathrm{rod}}}{V}
$$

where $N_{c}$ is as defined in Eq. (3), $V_{1 \text { rod }}$ is the volume of one rod, $V$ is the total simulation volume. Fits of their simulated data found that $B_{c}$ is 1.2 in the slender-rod limit, where

$$
\phi_{c, \text { iso }}^{\text {contact }} \cong \frac{0.60}{L / D} .
$$

This modification of the excluded volume model shows better agreement with our simulations than the purely analytical version of the excluded volume model (Table I). These comparisons of the isotropic systems demonstrate the necessity of simulating networks with finite $L / D$, which are not well described by traditional percolation models.

In addition to aspect ratio, particle orientation impacts the percolation threshold in networks of rods. ${ }^{5}$ Three examples of simulated morphologies with different $S$ values are shown in Fig. 1(a). At various fixed degrees of axial orientation, $S$, the critical volume fractions were determined from the calculated electrical conductivities using the power law in Eq. (1) [Fig. 1(b)]. As discussed above, the percolation threshold is higher for smaller $L / D$ in the isotropic network, $S=0$. The simulation results also show that the critical volume fractions for the smaller aspect ratio rods are more sensitive to axial orientation. For example, the percolation threshold approximately doubles as the axial orientation parameter increases from 0 to $\sim 0.9$ for the $L / D=10$ rods.

For comparison, Balberg's adaptation ${ }^{3}$ of the excluded volume model is shown in Fig. 1(b), which makes the simplifying assumption that

$$
\langle|\sin \gamma|\rangle \approx 1.25\langle|\sin \theta|\rangle,
$$

where $\langle|\sin \theta|\rangle$ is the two-dimensional average of the sine of the angle between one rod and a second rod parallel to the orientation axis. By calculating a normalized Gaussian distribution in $\sin \theta$, the resulting calculation yields $\phi_{c}^{\text {exvol }}$ as a function of $S$. As is evident from Fig. 1(b), the excluded volume model substantially underpredicts the critical volume fraction at low aspect ratio. This is an expected trend because the slender-rod limit assumption inherent in the excluded volume calculations underestimates the critical volume fraction for low aspect ratio fillers. More importantly, at $L / D$ $=10$, the excluded volume model shows a weaker dependence on the axial orientation than is found in our simulations. This increased discrepancy between the excluded volume model and our simulations for anisotropic networks is significant because particles of even modest aspect ratio are known to align in electromagnetic and flow fields. Our simulations can be used to determine $\phi_{c}$ as a function of $L / D$ and $S$ in the $L / D \leq 80$ regime, in which the accuracy of the excluded volume model breaks down and which encompasses many experimentally useful fillers.
Now we move to the main focus of our work, namely, the simulation of the electrical conductivity of percolated networks of rodlike particles as a function of axial alignment. To date, the morphology of percolating networks in terms of filler size, aspect ratio, concentration, and orientation has been the objective of theoretical and simulation approaches to percolation. ${ }^{1-11}$ Here, we have constructed percolating morphologies and used these morphologies to predict a physical property, specifically, electrical conductivity. This extension provides greater integration of simulations with experimental studies, particularly, in work with nanocomposites and nanoparticles.

At fixed concentrations above $\phi_{c, \text { iso }}$, we have simulated the morphology of composites containing rods with various aspect ratios and degrees of axial orientation, $S$. Figure 2 presents the calculated electrical conductivity from these simulated morphologies as a function of $S$. Notice first that at fixed $L / D$ and $S$, the electrical conductivity increases with increasing filler volume fraction as more conductive pathways form. In addition, the axial orientation of the rods alone is sufficient to change the electrical conductivity of the simulated networks by many orders of magnitude.

There are two characteristic trends in conductivity as a function of network orientation. First, at a fixed volume fraction and $L / D$, the electrical conductivities exhibit a substantial drop with increasing axial alignment. As the rods become highly aligned, the network structure is destroyed, causing the electrical conductivity to decrease. Second, in the range of $S<0.2$, for a fixed concentration and $L / D$, the simulations show a small maximum in conductivity. This maximum in electrical conductivity provides insight into the electrical conductivity in nanocomposites containing cylindrical fillers. The conductivity of our simulated systems relies on both the presence of at least one percolated pathway and the number of contacts (i.e., series resistance) within the pathway(s). Therefore, at $\phi>\phi_{c}$, electrical conductivity can increase only when (i) more percolated pathways form (causing a decrease in the total parallel resistance) or (ii) the series resistance (i.e., number of rod contacts) of the percolated pathways decreases. Because the maximum in electrical conductivity occurs at low degrees of orientation, the more likely explanation is that as the average axial orientation of each rod increases, the intersection probability of adjacent rods decreases ${ }^{11}$. This ultimately results in a decrease in the number of rod contacts in each percolated pathway, causing a slight maximum in the simulated electrical conductivity. As mentioned above, at higher degrees of orientation, all of the electrically conductive pathways are eventually destroyed, resulting in a loss of electrical conductivity. The structure of the percolated networks as a function of rod orientation is a topic of ongoing study.

The dramatic drop in electrical conductivity as a function of the axial orientation parameter can be considered as an orientation threshold. The critical axial orientation parameter, $S_{c}$, is the orientation above which all of the percolating clusters are destroyed even at volume fractions well above $\phi_{c \text {,iso. }}$. At $S<S_{c}$, the system is percolated and the conductivity is finite, but at $S>S_{c}$ the system is not percolated and the conductivity drops to 0 . As with the critical volume fraction, a power law defines the critical axial orientation parameter, $S_{c}$, 

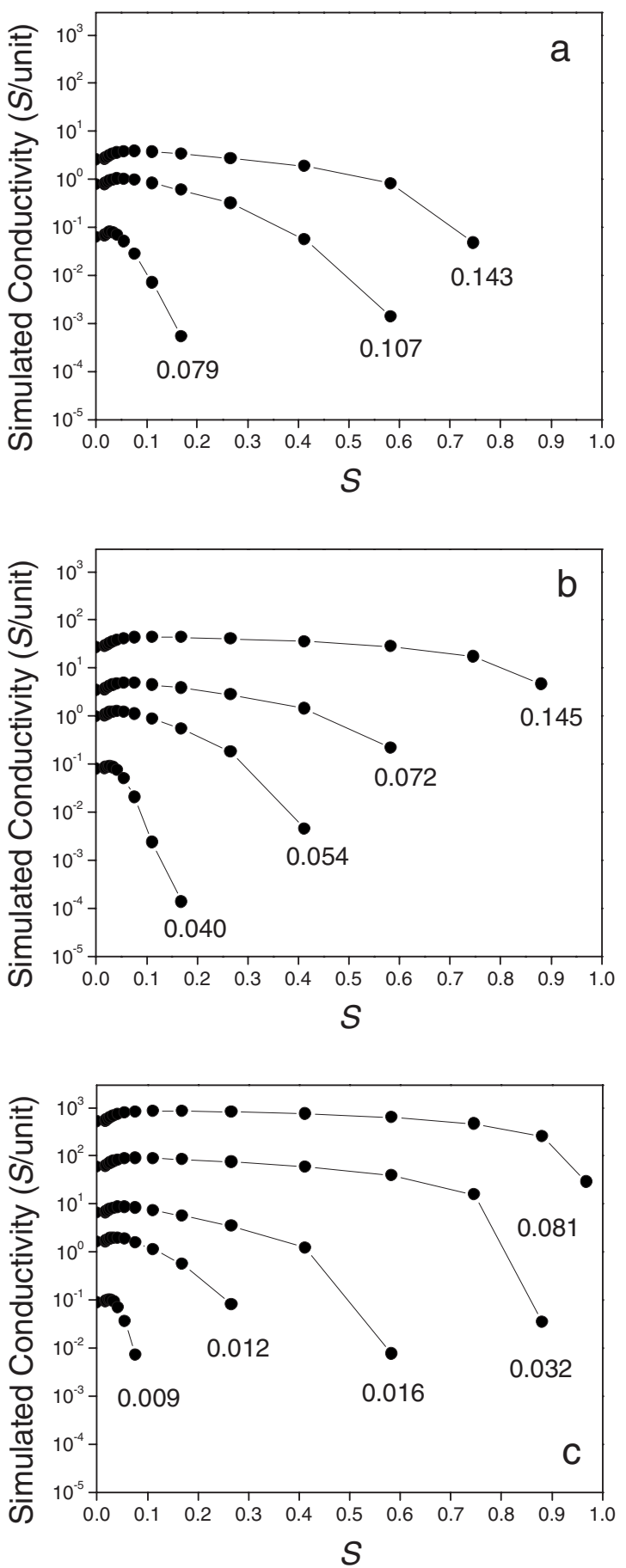

FIG. 2. Simulated electrical conductivity (Siemens/unit) vs $S$ for (a) $L / D=10$, (b) 20, and (c) 80 . The volume fraction, $\phi$, is given for each curve.

$$
\sigma_{\mathrm{el}} \propto\left(\frac{S_{c}-S}{S_{c}}\right)^{\alpha} .
$$

Figure 3 shows $S_{c}$ as a function of volume fraction. Consider a network of rods of fixed aspect ratio, wherein the volume fraction of rods, $\phi$, and the orientation of those rods, $S$, are independently controlled. When $(\phi, S)$ falls below the $S_{c}$ data in Fig. 3 for the specified aspect ratio, the resulting network
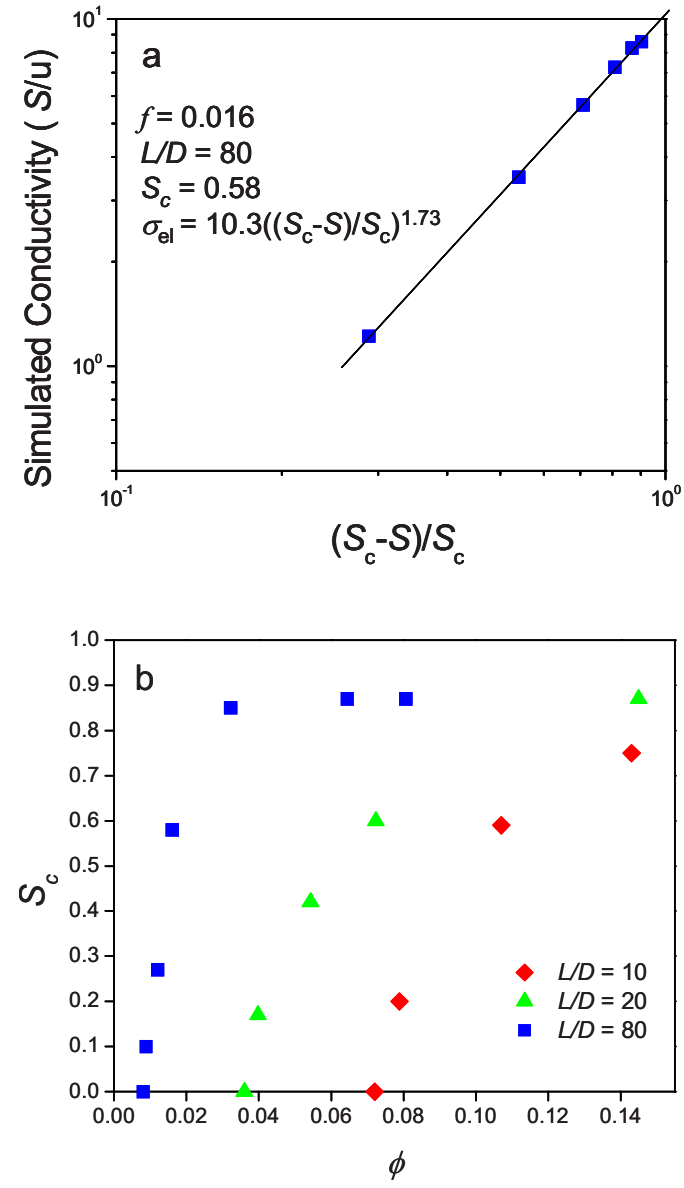

FIG. 3. (Color online) (a) The critical axial orientation parameter, $S_{c}$, was determined at fixed concentration and $L / D$ using a power law to relate electrical conductivity and the axial orientation parameter, $S$ [Eq. (8)]. (b) $S_{c}$ as a function of volume fraction of rods for three values of $L / D$. At $S=0$, the points correspond to $\phi_{c \text {,iso }}$ in Table I.

of rods has a high electrical conductivity. In contrast, when $(\phi, S)$ lies above the $S_{c}$ data, the electrical conductivity drops toward zero. The higher aspect ratio rods have a wider range of $\phi$ and $S$ over which electrical conductivity is high. Conversely, when the rods have $L / D=10$, significantly higher loadings are necessary before a well-aligned network provides good electrical conductivity. In practical terms, while lower aspect ratio rods might be less likely to align, smaller degrees of alignment are required to disrupt the percolated systems containing lower aspect ratio rods. Thus, regardless of the aspect ratio and volume fraction of rodlike conductive particles, the extent of axial orientation plays an integral role in determining the electrical conductivity.

Before concluding, we compare our simulation results with recent electrical conductivity measurements of polymer nanocomposites containing single-walled carbon nanotubes (SWCNTs). The effects of carbon nanotube orientation on electrical conductivity in polymer composites have been observed in doctor-bladed composites of multiwalled carbon nanotubes (MWCNTs) and cross-linked poly(furfuryl alcohol $)^{12}$ and in stretched composites of SWCNT and poly(methyl methacrylate) (PMMA). ${ }^{13}$ These composites exhib- 


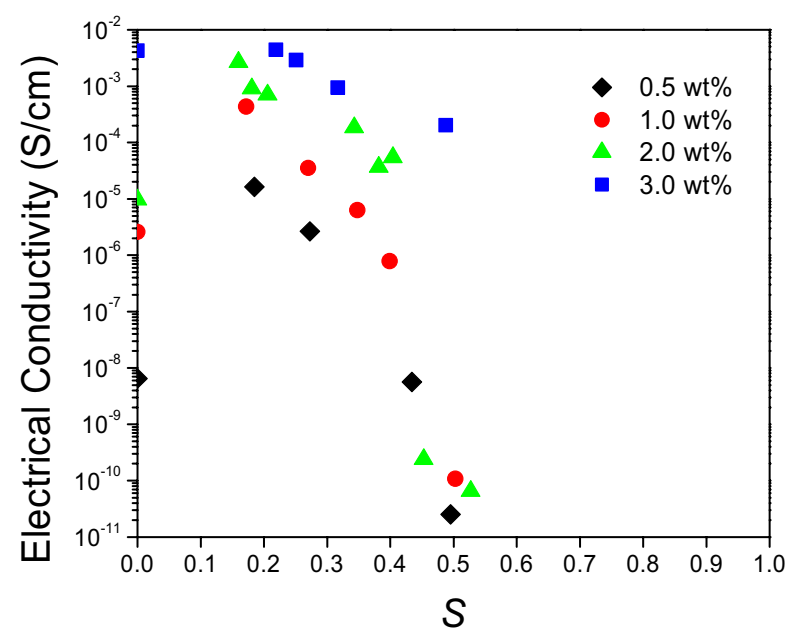

FIG. 4. (Color online) Experimental results by Du et al. (Ref. 5) from uniaxially aligned nanocomposite of SWCNT in poly(methyl methacrylate). The electrical conductivity has been replotted at fixed loading as a function of $S$. The nanotube bundles in these composites have $L / D \sim 45$ and $S$ was measured using an x-ray scattering method.

ited anisotropic electrical conductivity, with different values parallel and perpendicular to the alignment direction. In both of these materials, the conductivity parallel to the direction of nanotube alignment exceeded that perpendicular to it at all filler concentrations. However, a separate study found that melt-spun MWCNT/polycarbonate composites had immeasurably low electrical conductivity along the alignment direction at all experimental draw ratios. ${ }^{14}$ Although none of these papers quantified the nanotube orientation, it is clear that the alignment of conductive cylindrical particles in a nonconductive matrix is crucial for determining composite properties.

We compare our simulations more satisfyingly with a study by Du et al. ${ }^{5}$ of SWCNT/PMMA composites with filler orientations ranging from isotropic to significant uniaxial alignment. Alignment of the filler in the composites was quantitatively determined using small-angle x-ray scattering and reported as an azimuthal full width at half maximum. We have replotted their data in terms of the axial orientation parameter, $S$ (Fig. 4). The isotropic percolation threshold for these composites is reported to be $0.37 \mathrm{wt} \%$. At a fixed $S$ $\sim 0.3$, the electrical conductivity increases by $\sim 10^{3}$ as the SWCNT loading increases from 0.5 to $3.0 \mathrm{wt} \%$. As in our simulations, the most striking feature of these composites is that as the extent of alignment increases at a fixed concentration, there is a dramatic drop in electrical conductivity.
Also apparent in Fig. 4 is the presence of a maximum in electrical conductivity at slight uniaxial filler alignment, which becomes less pronounced with increasing SWCNT concentration. A more quantitative comparison between our simulations and these experimental results is unwarranted because the SWCNT bundles vary significantly in diameter, length, and electrical conductivity, in contrast to the monodisperse rods in our simulations. Du et al. ${ }^{5}$ also used a twodimensional simulation to explore the importance of orientation, but their simulations have been shown to suffer from finite-size effects.

\section{CONCLUSIONS}

We have simulated networks of soft-core, rod-shaped particles in three dimensions as a function of the number of rods, aspect ratio, and rod orientation as defined by the axial orientation parameter, $S$. Using the random network resistor model to mimic conductive particles in an insulating matrix, the electrical conductivities were determined and compared with earlier experimental results. At a fixed volume fraction and $L / D$, the calculations and experiments exhibit a critical orientation, $S_{c}$, above which the electrical conductivity decreases dramatically. Our simulations show that $S_{c}$ increases with both filler concentration and aspect ratio. Also, at fixed volume fraction and $L / D$, the electrical conductivity shows a maximum in both simulations and experiments, which is less pronounced at higher volume fractions and aspect ratios. These simulations are important because they calculate electrical conductivity for three-dimensional simulated samples containing rods of modest aspect ratio, with concentrations above the percolation threshold, and oriented over a wide range of axial alignments. This approach of simulating the morphology and calculating the electrical conductivity could be used as a predictive tool to design the optimal filler concentration and degree of filler orientation required to maximize electrical conductivity in polymer nanocomposites with conductive cylindrical fillers of finite dimension. Finally, the results of our simulations suggest that experimentalists reporting electrical conductivities in systems containing anisotropic particles should report not only the particle loading but also the filler aspect ratio and orientation, because all three attributes significantly impact the conductivity.

\section{ACKNOWLEDGMENTS}

S.I.W. acknowledges financial support from the GRFP at the National Science Foundation. K.I.W. acknowledges financial support from the Materials Research Science and Engineering Center, National Science Foundation (Grant No. 05-20020).

\footnotetext{
*winey@ seas.upenn.edu

${ }^{1}$ S. Kirkpatrick, Rev. Mod. Phys. 45, 574 (1973).

${ }^{2}$ I. Balberg and N. Binenbaum, Phys. Rev. B 28, 3799 (1983).

${ }^{3}$ I. Balberg, C. H. Anderson, S. Alexander, and N. Wagner, Phys. Rev. B 30, 3933 (1984).
}

${ }^{4}$ A. Behnam, J. Guo, and A. Ural, J. Appl. Phys. 102, 044313 (2007).

${ }^{5}$ F. Du, J. E. Fischer, and K. I. Winey, Phys. Rev. B 72, 121404(R) (2005).

${ }^{6}$ L. Berhan and A. M. Sastry, Phys. Rev. E 75, 041120 (2007). 
${ }^{7}$ A. L. R. Bug, S. A. Safran, and I. Webman, Phys. Rev. Lett. 54, 1412 (1985).

${ }^{8}$ A. L. R. Bug, S. A. Safran, and I. Webman, Phys. Rev. B 33, 4716 (1986).

${ }^{9}$ Z. Néda, R. Florian, and Y. Brechet, Phys. Rev. E 59, 3717 (1999).

${ }^{10}$ M. Foygel, R. D. Morris, D. Anez, S. French, and V. L. Sobolev, Phys. Rev. B 71, 104201 (2005).
${ }^{11}$ S. H. Munson-McGee, Phys. Rev. B 43, 3331 (1991).

${ }^{12}$ L. J. Lanticse, Y. Tanabe, K. Matsui, Y. Kaburagi, K. Suda, M. Hoteida, M. Endo, and E. Yasuda, Carbon 44, 3078 (2006).

${ }^{13}$ J. Dai, Q. Wang, W. Li, Z. Wei, and G. Xu, Mater. Lett. 61, 27 (2007).

${ }^{14}$ P. Pötschke, H. Brünig, A. Janke, D. Fischer, and D. Jehnichen, Polymer 46, 10355 (2005). 